\title{
A microstructural study of superconductive nanocrystalline diamond
}

\author{
M.P. Villar ${ }^{* 1}$, Ma.P. Alegre ${ }^{1}$, D. Araujo ${ }^{1}$, E. Bustarret ${ }^{1,2}$, P. Achatz $^{2}$, L. Saminadayar ${ }^{2}$, C. \\ Bauerle $^{2}$ and O.A. Williams ${ }^{3}$ \\ ${ }^{1}$ Dept. Ciencia de los Materiales e IM y QI. Universidad de Cádiz. Campus Universitario Río San Pedro. \\ 11510-Puerto Real (Cádiz). Spain \\ 2 Institut Néel, CNRS-UJF. 25 av. des Martyrs, 38042-Grenoble. France \\ ${ }^{3}$ Fraunhofer Institut Angewandte Festkörperphysik, Friburg, Germany \\ Received ZZZ, revised ZZZ, accepted ZZZ \\ Published online ZZZ
}

PACS 81.07.Bc 68.37.Lp 68.37.Og 74.78.Na

\begin{abstract}
A transmission electron microscopy (TEM) study of superconducting nanocrystalline diamond (NCD) continuous layers is reported. The high resolution transmission electron microscopy (HREM) and the diffraction contrast modes of observations are used to reveal the nanograins configuration. Three types of them are observed: First, close to the interface with the $\mathrm{Si} / \mathrm{SiO}_{2}$ substrate, 10 to $20 \mathrm{~nm}$-sized diamond seeds resulting from the $5 \mathrm{~nm}$ size diamond powder deposition before growth that show some regrowth during CVD process, second a diamond overgrown layer, quasi-epitaxially by coalesced columnar NCD grains, and finally, up to the free surface, a thin disordered region composed of nanocrystallites smaller than $6 \mathrm{~nm}$. This last layer was not nominally expected and is attributed to a renucleated-like (RND) diamond layer embedding ultra nanocrystalline grains. Diffraction contrast observations confirm this HREM observed behaviour.
\end{abstract}

.

1 Introduction

Despite recent advances in manufacturing and enlarging synthetic crystalline diamond substrates which open a practicable route for growing epitaxially all the layers necessary for building electronic devices, the principal bottleneck to industrially implant such devices is the substrate growth area and price. Heteroepitaxial growth by microwave plasma-enhanced chemical vapour deposition (MPCVD), in particular on large size Si-related substrates, can be an alternative to homoepitaxy in some cases, and yield either textured large grain free-standing thick films or thin nanocrystalline (NCD) or ultrananocrystalline (UNCD) layers of diamond. Controlled doping by boron from traces close to ppb concentrations up to the atomic percent range has by now been established as an efficient way to tailor the electrical properties of diamond, thanks to the acceptor level of substitutional boron, which lies 0.37 $\mathrm{eV}$ above the valence band maximum. In particular, above a boron concentration around 2.5 to $4.510^{20}$ $\mathrm{cm}^{-3}$ depending on the nanocrystalline [1] or single crystal [2] nature of diamond, the films become truly metallic, i.e. they preserve a finite resistivity value as the temperature is decreased toward zero.

Actually, when the temperature is low enough, virtually all of these metallic films become superconducting, as demonstrated first for polycrystalline samples grown by High Pressure and High Temperature [3], then for MPCVD-grown single crystal epilayers [4] and later for nanocrystalline films $[1,5]$. Boron doping-induced superconductivity in other group IV semiconductors such as silicon [6,7] and $\mathrm{SiC}[8,9]$ has also been reported recently, but diamond remains by far the most intensively studied of these covalent superconductors [10]. In all cases, there has been some concern about boron incorporation in sites different from the ideal isolated substitutionnal site. However, apart from some experimental

Corresponding author: e-mail: pilar.villar@uca.es, Phone: +00 34956016 348, Fax: +00 34956016337 
studies of boron pairing [11], clustering [12], boron passivation by hydrogen [13] or interstitial incorporation [14] effects, the influence of the crystallinity of the diamond lattice on the superconductive properties has not been yet investigated in full detail, in particular for the micro- or nanocrystalline material. The aim of the present ongoing study is thus to contribute to discuss the superconductivity in polycrystalline diamond on the basis of a preliminary microstructural study of boron-doped CVD nanocrystalline diamond using transmission electron microscopy (TEM) techniques.

\section{Experimental}

Heavily boron-doped diamond layers, with nominal thicknesses of ca. $200 \mathrm{~nm}$ and $130 \mathrm{~nm}$ for sample \#118 and \#123 respectively, were grown by MPCVD onto Si substrates with an intermediate $\mathrm{SiO}_{2}$ buffer layer, seeded by diamond nanoparticles in an ultrasonic bath [15]. Two sets of samples were grown, labeled \#118 and \#123. Growth temperature was $700^{\circ} \mathrm{C}$ and methane was diluted (3\%) in hydrogen while the trimethylboron (TMB) to methane molar ratio was fixed to obtain a boron atomic concentration in the $10^{21} \mathrm{~cm}^{-3}$ range. Growth was performed for $25 \mathrm{~min}$ for sample \#118 and for $35 \mathrm{~min}$ for sample \#123 at total pressures of 47 and 40 Torr, respectively.

TEM studies are performed on a Jeol 1200EX $(120 \mathrm{keV})$ for the diffraction contrast observations and on a Jeol 2010F (200 keV) for the HREM observations. Cross-sectional and planar view preparations were carried out by standard mechanical thinning followed by ion milling (Gatan PIPS). Electrical measurements were carried out at low temperatures with and without a magnetic field (superconducting coil) using a four-point Van der Pauw geometry.

\section{Results and discussion}

Samples \#123 and \#118 are shown on Fig. 1a to become superconducting (zero resistance) below 0.4 and $1.6 \mathrm{~K}$ respectively. This figure illustrates also the fact that the critical field $\mathrm{Hc} 2$ of this type II superconductor can reach relatively high values, of the order of $3 \mathrm{~T}$ around $200 \mathrm{mK}$ for sample \#118. In the following, two working modes of the TEM were used to show different features of the polycrystalline diamond configuration. High resolution TEM (HREM) mode allows to observe the atomic planes by choosing some diffracted electron beams and then reconstructing the image in the microscope by interference. Diffraction contrast mode (CTEM) allows to reveal crystal defects and, in the present case, grain orientations by choosing one among the diffracted beams.

All the studied superconductive samples have the same general structure, that is, a first set of small diamond grains (ca. $20 \mathrm{~nm}$ ) over the $\mathrm{Si} / \mathrm{SiO}_{2}$ substrate, followed by a set of larger and columnar diamond crystals $(30-60 \mathrm{~nm})$. Above those geometrically well defined grains, the whole structure is covered by an apparentely disordered capping layer. Differences between both samples lie in the thickness of the different layers, mainly in the disordered capping layer, thicker in the first one (50-120 nm in \#118 vs. 20-30 $\mathrm{nm}$ in sample \#123).

Figure 1b shows the general features of sample \#123. As can be seen, a layer of nanosized crystalline seeds (see dashed circles) extends over the $\mathrm{SiO}_{2}$-diamond interface. This layer was not too much misoriented with respect to the larger grains grown afterwards. Indeed, no contrasts were observed by the diffraction contrast mode of the TEM (see Figs. 2a and 2c). Clear evidences of this nanosized crystalline seed layer are obtained by HREM, as shown in Fig. 1b, where a cross sectional view of the same sample is presented (see dashed circles).

These seeds are not so well revealed by CTEM. Figures $2 \mathrm{a}$ and $2 \mathrm{c}$ evidence the second columnar grown grains. The dashed circle represents the location and the extension of the selected area aperture used to record the SAED (select area electron diffraction) pattern shown in the inset. The polycrystalline character is clearly revealed by the small spots aligned around a ring in the diffraction pattern. As can be observed in it, rings are formed by brighter and larger spots and lighter and smaller ones, marked in the inset with red and blue circles, respectively. The brighter spots can be attributed to the larger diamond crystals observed in Fig. 2c micrograph. To confirm this point, Fig. 2c contains the micrograph from the image built selecting the spot indicated by the red circle in Fig. 2a, and the micrograph undoubtely shows 
(a)

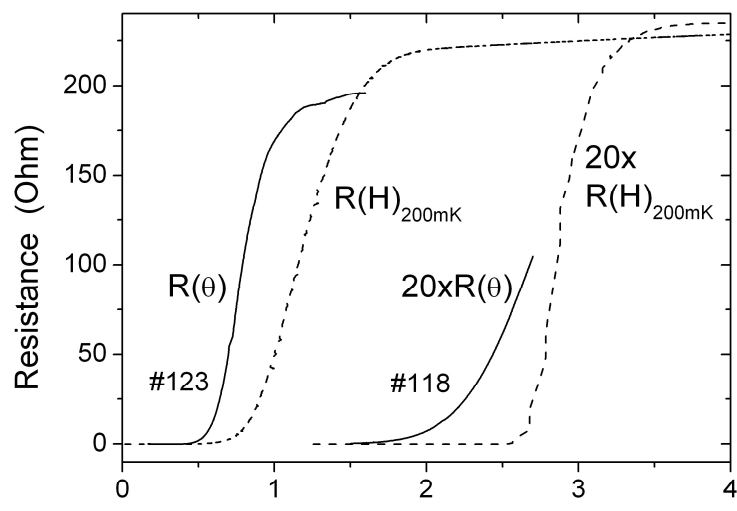

Temperature $\theta(\mathrm{K})$ or Magnetic Field $\mathrm{H}(\mathrm{T})$

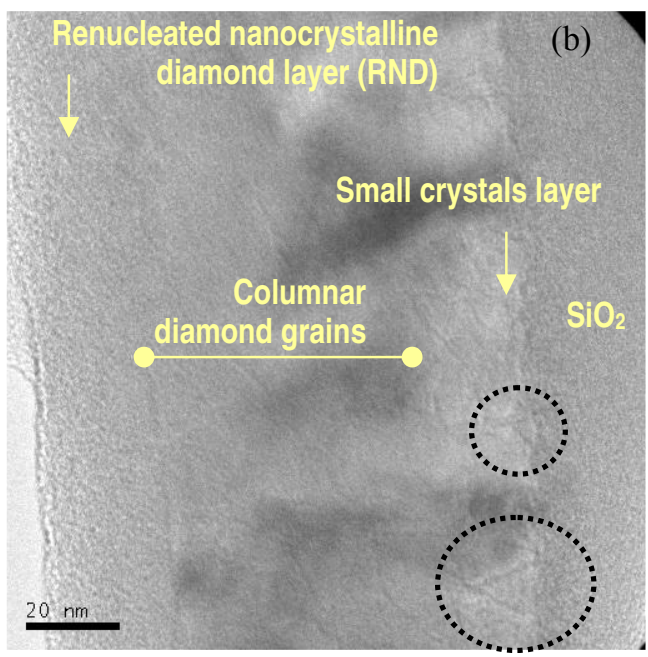

Fig.1 (a) Electrical resistance of samples \#118 and \#123 as a function of temperature (solid lines) without any applied magnetic field, and as a function of the magnetic field (broken line) at a fixed temperature of $200 \mathrm{mK}$. The resistance values of sample \#118 have been multiplied by 20. (b) Diamond layer observed in HREM where three type of crystals can be distinguished in sample \#123 : (i) $20 \mathrm{~nm}$ sized grain when the growth begins at the $\mathrm{SiO}_{2} / \mathrm{diamond}$ interface (see dashed circles), (ii) larger grains that were revealed also in the diffraction contrast observations, (iii) a 20-30 nm thick superficial layer that seems here to have an amorphous character but shown to include ultrananocrystals of diamond. The two micrographs are recorded on two vicinal regions, as can be recognized by the green circle region

From SAED analysis, the average lattice parameter of diamond, corresponding to all the diamond grains present in the structure, is estimated to amount values near $3.58( \pm 0.01) \AA$. This confirms the high doping level of the grown grains if this value is compared to previously published lattice parameter vs. doping curves $[16,17]$. Indeed, those authors reported values ranging from 3.566 to $3.578 \AA$ for undoped to $1 \%$ at. boron doping respectively.

Going back to the bright contrast of Fig. $2 b$ justified by diffraction of some sort of very tiny crystals in the amporphous capping layer, HREM is carried out to clarify this point. Detailed HREM and diffraction contrast analysis of this layer for both samples reveal the nucleation of very small diamond grains, with 3-6 nm size. These crystals are found as embebded small periodical organizations in the matrix, showing random orientations, and rather look an incipient organization of the matter in this capping layer. This very particular characteristic can be observed in the two superconductive samples under study, but not in other non-superconductive similar samples [18]. The HREM micrograph of Fig. 3 evidences these very small crystals. Two of them are chosen (see dashed circles on Fig. 3b), as an example, to show their periodical arrangement by means of line profiles. As the nano-sized crystals are not oriented in the same 

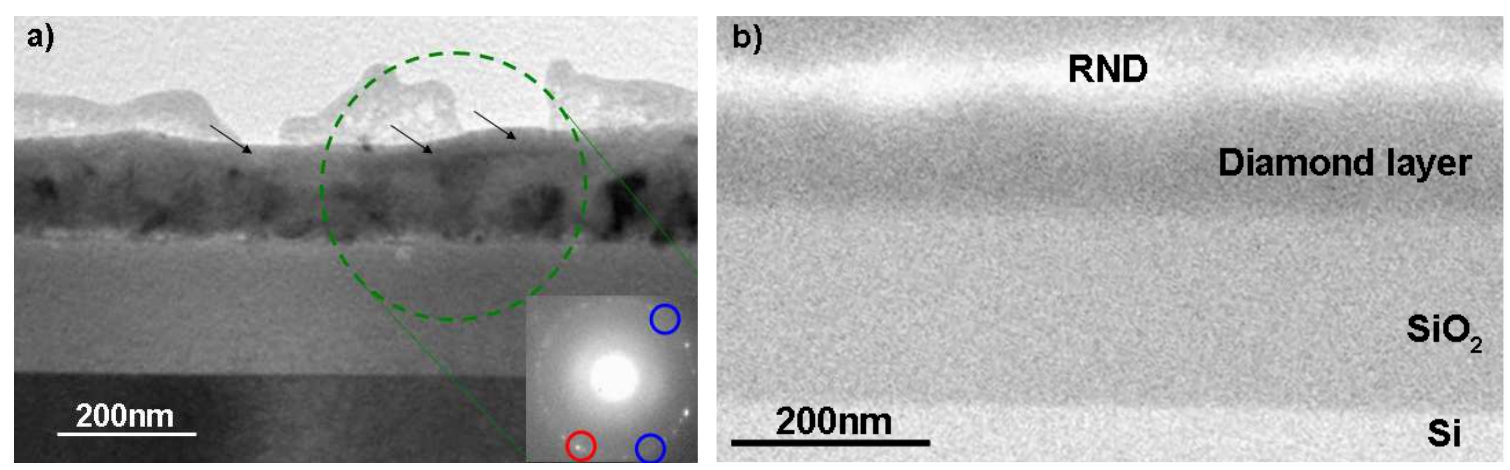

direction, periodicities of the fringes vary between those just nucleated entities. As the periodicity is more than 3 fringes, it is usually accepted that they correspond to some crystalline entities. Fig.3a and $b$ indicate that nanoentities, with a lattice parameter of $3.58( \pm 0.01) \AA$ (deduced from the SAED pattern) are present in that layer. Thus we deduce that those clusters correspond to diamond nanoparticles. From the growing specifications, this layer should not exist, therefore we attribute those diamond nanocrystals to a renucleation of nanodiamond crystals (RND). Preliminar HAADF experiments and lattice parameter values obtained from SAED patterns seems to indicate a highly boron dopinng of this RND layer.

Fig.2: (a) Micrograph of one region of the sample \#123, where the grain orientation were studied by diffraction contrasts. In the inset the SAED corresponding to the green circle area. (b) Micrograph recorded with the blue circle aperture locations. (c) Micrograph obtained by locating the diffraction contrast aperture on the red circle place. The red rectangle show the area corresponding to Fig.2a.

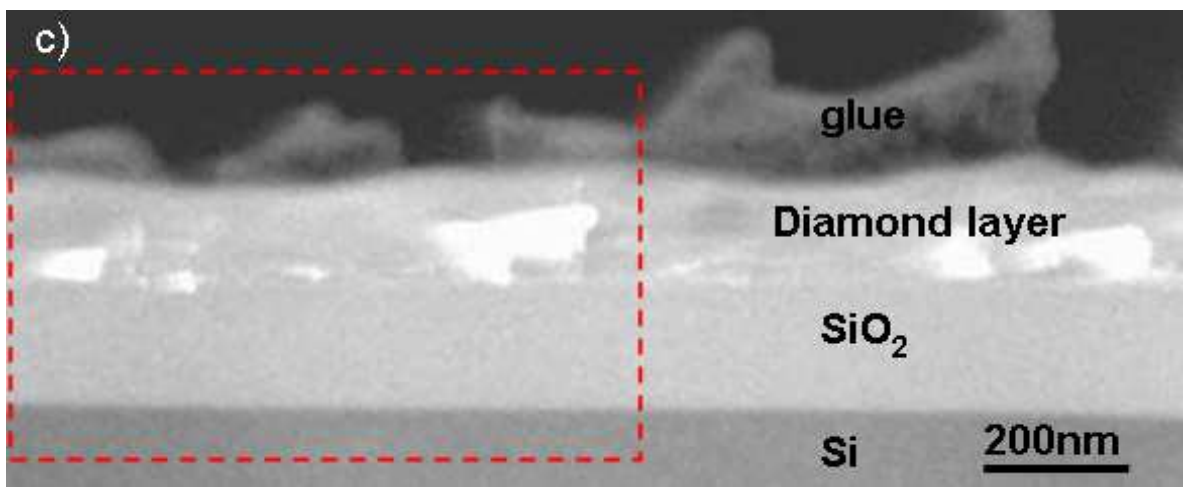

Comparisons between samples of this study and similarly grown non-superconductive samples [18] have been established to understand both the origin and the role of this RND layer. The latter is only observed in the superconductive samples, although setting a clear relation between the disordered capping layer with the embebded ultrananocrystals RND layer and the superconductivity behaviour of poly/nanocrystalline heavily boron doped diamond definitively requires definitively deeper studies. It will be necessary to confirm that the RND layer is not the result of some artifact due to reaction of residual $\mathrm{CH}_{4}$ gas and TMB during the growth experiment shutdown. However, in such case, it should also appear in the non-superconductive samples, as they were grown with the same equipment and under similar conditions. 
As there are very few reported results concerning structural or microstructural studies of superconductive diamond, this point should be studied in more depth. On the other hand, investigations to understand the origin of such RND layer are ongoing.
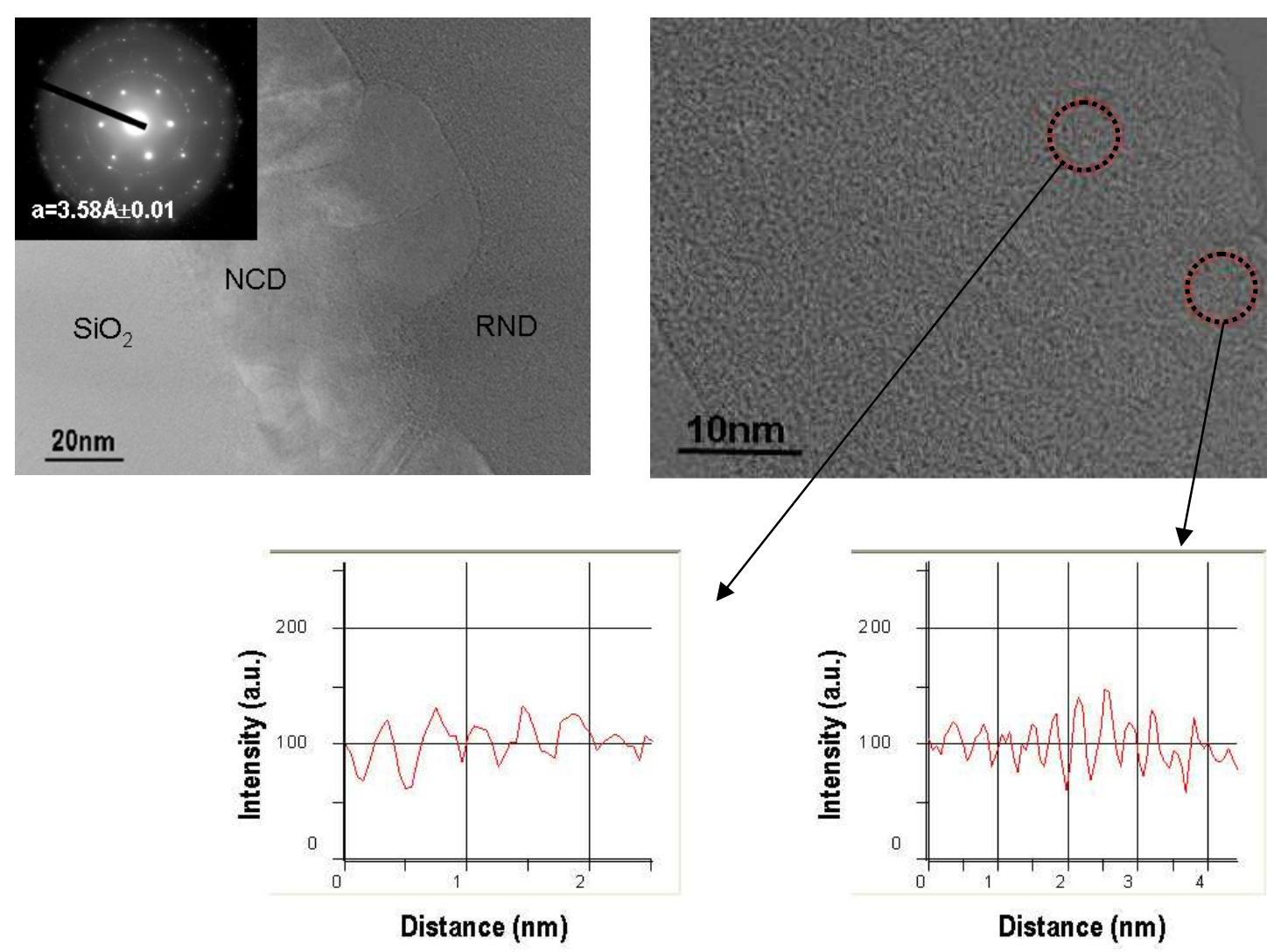

Fig. 3 HREM analysis of sample \#118. a) Again, a vision of the whole structure of sample \#118 showing similar features: first small grains followed by larger ones, and covered by the amorphous capping layer of about $60-120 \mathrm{~nm}$ thick. b) Detail of the capping layer. Carefull analysis reveals the presence of very small and incipient formation of ultra-nanocrystals (around 3-6 nm). Line profiles of such ultra-nanocrystals showing a periodical organization of matter are also included.

\section{Conclusions}

A TEM study of superconducting heavily boron doped $\mathrm{Si} / \mathrm{SiO}_{2} /$ diamond structures is presented. Three types of grain configurations have been revealed using CTEM and HREM modes of the microscope: (i) first diamond seeds of $20 \mathrm{~nm}$ diameter on the $\mathrm{SiO}_{2}$ layer allow to nucleate the diamond layer, (ii) larger diamond grains are revealed with sizes around $50 \mathrm{~nm}$ corresponding to NCD and finally (iii) RND grains embedded in a superficial layer are shown by both HREM and CTEM. More studies to determine if the latter is responsible in some way for the superconductive character of those samples are imperatively required. 
Acknowledgements We thank J.M. Geraldía for technical support with HREM and HAADF measurements. All microscopy work has been carried out at the Electron Microscopy Facilities of the University of Cádiz (Central Services of Science and Technology). This study was supported by regional funding from the Andalusian Council (Junta de Andalucía, Spain).

\section{References}

[1] W. Gajewski, O.A. Williams, P. Achatz, K. Haenen, E. Bustarret, M. Stutzmann and J.A. Garrido, Phys. Rev. B 79, 45206 (2009).

[2] T. Klein, P. Achatz, J. Kacmarcik, C. Marcenat, F. Gustafsson, J. Marcus, E. Bustarret, J. Pernot, F. Omnès, Bo E. Sernelius, C. Persson, A. Ferreira da Silva and C. Cytermann, Phys. Rev. B 75, 165313 (2007).

[3] E.A. Ekimov, V.A. Sidorov, E.D. Bauer, N.N. Mel'nik, N.J. Curro, J.D. Thompson and S.M. Stishov, Nature 428, 542 (2004).

[4] E. Bustarret, J. Kačmarčik, C. Marcenat, E. Gheeraert, C. Cytermann, J. Marcus and T. Klein, Phys. Rev. Lett 93, 237005 (2004).

[5] M. Nesladek, D. Tromson, C. Mer, P. Bergonzo, P. Hubik and J.J. Mares, Appl. Phys. Lett. 88, 232111 (2006).

[6] E. Bustarret, C. Marcenat, P. Achatz, J. Kačmarčik, F. Lévy, A. Huxley, L. Ortéga, E. Bourgeois, X. Blase, D. Débarre and J. Boulmer, Nature 444, 465 (2006).

[7] D. Cammilleri, F. Fossart, D. Debarre, C. Tran Manh, C. Dubois, E. Bustarret, C. Marcenat, P. Achatz, D. Bouchier and J. Boulmer, Thin Solid Films 517, 75 (2008).

[8] Z.-A. Ren, J. Kato, T. Muranaka, J. Akimitsu, M. Kriener and Y. Maeno, J. Phys. Soc. Jap 76, 103710 (2007).

[9] M. Kriener, Y. Maeno, T. Oguchi, Z.-A. Ren, J. Kato, T. Muranaka and J. Akimitsu, Phys. Rev. B 78, 024517 (2008).

[10] X. Blase, E. Bustarret, C. Chapelier, T. Klein and C. Marcenat, Nature in press, (2009).

[11] E. Bourgeois, E. Bustarret, P. Achatz, F. Omnès and X. Blase, Phys. Rev. B 74, 094509 (2006).

[12] N. Dubrovinskaia, R. Wirth, J. Wostnitza, T. Papageorgiou, H.F. Braun, N. Miyajima, L. Dubrovinsky, PNAS 105, 11619 (2008)

[13] H. Mukuda, T. Tsuchida, A. Harada, Y. Kitaoka, T. Takenouchi, Y. Takano, M. Nagao, I. Sakaguchi, T. Oguchi and H. Kawarada, Phys. Rev. B 75 , 033301 (2007).

[14] Y. Kato, F. Matsui, T. Shimizu, T. Matsushita, F.Z. Guo, T. Tsuno and H. Daimon, Sci. Technol. Adv. Mater. 7, S45 (2006).

[15] O.A. Williams, M. Nesladek, M. Daenen, S. Michaleson, A. Hoffman, E. Osawa, K. Haenen and R.B. Jackman, Diam. and Rel. Mater. 17, 1080 (2008).

[16] T.Wojewoda, P. Achatz, L. Ortéga, F. Omnès, C. Marcenat, E. Bourgeois, X. Blasé, F. Jomard and E. Bustarret, Diam. and Rel. Mater. 17, 1302 (2008).

[17] V.V. Brazhkin, E. A. Ekimov, A.G. Lyapin, S.V. Popova, A.V. Rakhmanina, S.M. Stishov, V.M. Lebedev, Y. Katayama and K. Kato, Phys. Rev. B 74, 140502 (R) (2006).

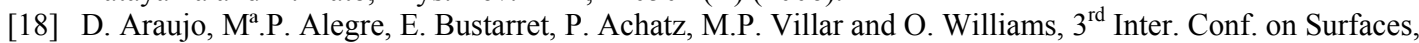
Coatings and Nanostructured Materials (NanoSMat), Barcelona, 21-24 Oct. (2008). 